\title{
Determinantes da satisfação no atendimento das Unidades Básicas de Saúde (UBS)
}

\author{
Determinants of satisfaction with the care provided by Basic Health \\ Units (BHU)
}

Maria Alice Vaz Gomes (https://orcid.org/0000-0002-5308-5541) ${ }^{1}$

Vinicius de Oliveira Pinto (https://orcid.org/0000-0001-6226-5651) ${ }^{1}$

Francisco Carlos da Cunha Cassuce (https://orcid.org/0000-0002-7545-3747) ${ }^{1}$
${ }^{1}$ Departamento de Economia, Universidade Federal de Viçosa. Av. Peter Henry Rolfs s/n, Campus Universitário. 36570000 Viçosa MG Brasil. mariaalicevazgomes@ hotmail.com

\begin{abstract}
The scope of this paper is to analyze the determinants of satisfaction with the care provided by Basic Health Units (BHU). The data were extracted from the external evaluation microdata of the National Program for Improvement of Access and Quality of Primary Care (PIAQ) for the year 2013. The results revealed that individuals in better financial standing tend to be less satisfied with the care provided by the UBS. In addition to this, evaluating variables such as waiting times for consultation, respect for the patient and the guarantee of privacy, it becomes clear that the improvement in the perception of care provided by the UBS can be achieved with better administration of resources, namely without increasing financial overheads. Lastly, it becomes apparent that individuals value the care provided more than the infrastructure of the UBS when asked to evaluate care.
\end{abstract}

Key words Satisfaction, BHU, Health determinants
Resumo Este trabalho analisa os determinantes da satisfação no atendimento das Unidades Básicas de Saúde (UBS). Os dados foram extraídos dos microdados de avaliação externa do Programa Nacional de Melhoria do Acesso e da Qualidade da Atenção Básica (PMAQ), do ano de 2013. Os resultados mostraram que indivíduos em melhor condição financeira tendem a ficar menos satisfeitos com o atendimento na UBS. Além disso, avaliando variáveis como tempo de espera para a consulta, o respeito ao paciente e a garantia de privacidade, é possivel indicar que a melhora na percepção dos atendimentos nas UBS pode ser alcançada com uma melhor administração dos recursos, ou seja, sem aumento de gastos financeiros. Por fim, pode-se afirmar que os indivíduos valorizam mais o atendimento do que a infraestrutura da UBS no momento de avaliar o atendimento.

Palavras-chave Satisfação, UBS, Determinantes de saúde 


\section{Introdução}

O Brasil é uma economia em desenvolvimento, marcada pela alta concentração de renda. De acordo com dados do IBGE ${ }^{1}$, em 2017 os 10\% mais ricos da população brasileira detinham $43,3 \%$ da renda total gerada no país, enquanto que os $10 \%$ menos abastados respondiam por apenas $0,7 \%$ dessa renda, o que indica uma ligeira piora quando comparado com o ano de 2013, quando os índices eram, respectivamente, $41,7 \%$ e $1,2 \%$. Tal concentração de renda influencia na tomada de decisão da população no que diz respeito ao planejamento familiar, visto que bens como alimentação são priorizados e outros, também considerados de primeira necessidade, tais como o consumo de serviços de saúde são preteridos.

Nesse contexto, a condição socioeconômica da grande maioria das famílias brasileiras fez com que as ofertas de serviços de saúde por parte do Estado fossem essenciais. Sendo assim, a Constituição Federal Brasileira de 1988 (CF-88) assegurou que "Saúde é um direito de todos e dever do Estado". Desta maneira, foi criado o Sistema Único de Saúde (SUS), um grande sistema de saúde pública que abrange atendimentos de pouca e de grande complexidade, garantindo o acesso integral, universal e gratuito a saúde para toda a população brasileira ${ }^{2}$.

Nesse complexo sistema, a Unidade Básica de Saúde (UBS) é o primeiro contato do indivíduo com o SUS e tem como função promover a atenção integral à saúde básica dos indivíduos, estando presente em locais de fácil acesso, o que a deixa próxima do cotidiano da população, desempenhando papel principal na garantia de acesso à saúde de qualidade.

Todo esse sistema público de saúde atravessa uma crise com unidades de atendimento sobrecarregadas, falta de materiais e infraestrutura. Segundo Pegoraro ${ }^{3}$, esta crise está atrelada ao fato de que o gasto total com saúde em porcentagem do PIB é pequeno em relação a países com sistema de saúde semelhante. Além disso, o escasso recurso, muitas vezes, é gerido de forma ineficiente piorando ainda mais os resultados do sistema.

Diante do exposto, analisar a satisfação dos usuários dos serviços prestados pelas UBSs seria uma forma de avaliar a qualidade e o alcance do atendimento, assim como a eficiência na alocação dos recursos dos contribuintes. Tal análise possibilitaria o direcionamento de ações capazes de melhorar a percepção do serviço oferecido.
Nesse sentido, Akerman e Nadanovsky ${ }^{4}$ destacaram a importância de se considerar a satisfação do usuário dos serviços de saúde na busca de oferta por um serviço de qualidade, ou seja, é impossível ter um serviço de qualidade se o usuário não estiver satisfeito.

Silva e Formigli ${ }^{5}$ e Espiridião e Trad ${ }^{6}$ também apontam os determinantes da satisfação com a saúde pública. Para os autores, há quatro principais determinantes dessa satisfação: características dos pacientes; características dos profissionais; aspectos da relação médico-paciente e fatores estruturais e ambientais.

Sendo assim, o objetivo deste trabalho é avaliar a satisfação do indivíduo atendido pelas UBS no Brasil considerando dados de 2013. Especificamente, pretende-se: verificar como o nível de satisfação da população é influenciado pela infraestrutura das UBS; analisar o impacto do atendimento e da disponibilidade de médicos sobre a percepção de satisfação da população atendida e avaliar como as características da população alteram o nível de satisfação com o serviço prestado pela UBS no Brasil.

Além dessa introdução, o trabalho apresenta uma revisão de literatura onde é definido o conceito de satisfação em saúde pública, assim como os seus principais determinantes, além dos mecanismos através dos quais esses argumentos afetam o nível de satisfação. Na seção III apresenta-se a metodologia utilizada para explicar o nível de satisfação da população brasileira além da fonte de dados. Na seção IV são apresentados os resultados e, por fim, na seção $V$ são apresentadas as considerações finais do estudo.

\section{Revisão de literatura}

A definição de satisfação é algo subjetivo, mas é possível indicar argumentos capazes de afetar o nível de satisfação de um indivíduo. Quando se trata do consumo de serviços de saúde pública pode-se determinar que a percepção da satisfação seria afetada pelas características individuais dos pacientes, pela administração no atendimento e pela infraestrutura da unidade que recebe o usuário.

Vaitsman e Andrade ${ }^{7}$ definem a satisfação em saúde através de um conjunto amplo e heterogêneo de pesquisas que visam saber a opinião dos usuários de serviços de saúde de um modo geral, englobando as distintas dimensões do cuidado à saúde, da relação médico-paciente até a infraestrutura das unidades de atendimento e a qualida- 
de dos profissionais e visa incorporar os pacientes na definição de parâmetros e mensuração da qualidade dos serviços oferecidos. Ao definirem satisfação em saúde como um conceito operacional que incorpora o ponto de vista e a expectativa do usuário, destacam importantes diferenças quando se avaliam serviços privados e públicos. No setor público, o usuário ora se coloca como consumidor, avaliando os serviços oferecidos a partir de seus ganhos individuais e ora se coloca como cidadão, avaliando os serviços oferecidos considerando os ganhos da sociedade como um todo. Ao considerar o setor privado, no que tange à escolha, o usuário tem a opção de procurar outro serviço ou outro profissional caso se sinta insatisfeito, o que é praticamente impossível no setor público.

Williams $^{8}$ afirma que a satisfação do usuário está relacionada ao papel que este se atribui em relação ao sistema público de saúde, em como ele entende seus direitos e deveres. Já Bernhart et al. ${ }^{9}$ citam que o setor público envolve aspectos mais complexos ao avaliar a satisfação do usuário, sendo o principal deles o sentimento de gratidão. Este aspecto é comum em países pobres e dificulta uma visão mais crítica. Neste caso, ainda há a influência da confiança da população no governo, nos políticos e nos servidores públicos.

Mais detalhadamente, os estudos existentes sobre satisfação em saúde pública apontam como principais determinantes a relação médico-paciente, aspectos relacionados à infraestrutura da UBS, acessibilidade, tempo de espera para ser atendido, aspectos relacionados aos profissionais que atuam nessas unidades e características referentes aos usuários. As variáveis citadas são pressupostas na expectativa e no desejo dos pacientes e em como eles percebem e avaliam a qualidade dos serviços oferecidos.

Moimaz et al..$^{10}$ avaliaram o grau de satisfação de usuários de saúde pública municipal em cinco cidades de pequeno porte do estado de São Paulo com características demográficas e socioeconômicas semelhantes, que têm a Estratégia de Saúde da Família implantada em seu sistema de saúde para o ano de 2006, através de entrevista com chefes de família dos municípios analisados. Os autores concluíram que a infraestrutura das unidades de atendimento afeta positivamente o nível de satisfação dos usuários.

Callou Filho et al. ${ }^{11}$ realizaram estudo sobre satisfação em relação à estratégia adotada para a saúde da família no município de Fortaleza para o ano de 2015, através de questionários aplicados em usuários acima de 18 anos que estavam pre- sentes na unidade no momento da coleta de dados e verificaram que a infraestrutura e o serviço prestado pelos profissionais possuem relação positiva com o nível de satisfação. Segundo os autores, os pacientes avaliam a infraestrutura da UBS de acordo com a limpeza, segurança, estrutura física das salas da UBS, assiduidade e remédios oferecidos pela unidade. Ao analisar que grande parte dos usuários entrevistados avaliaram esses aspectos como bons ou razoáveis é possível afirmar que o nível de satisfação aumenta se os usuários se sentem seguros ao recorrerem à UBS, se a manutenção da limpeza da unidade é feita com frequência e se estão confortáveis nas salas da UBS dada sua estrutura física.

Sá et al. ${ }^{12}$ analisaram o conhecimento e a satisfação sobre acolhimento em uma Unidade Básica de Saúde no município de Fortaleza, no ano de 2009, através de entrevistas semiestruturadas com usuários acima de 18 anos que estavam presentes na unidade no momento da coleta de dados. Os autores relataram como aspectos negativos o longo tempo de espera, principalmente nos serviços ambulatoriais e hospitalares, o não cumprimento de horários por parte dos trabalhadores das unidades e atitudes não profissionais, como a falta de assiduidade e a pontualidade dos profissionais. Muitas vezes, os usuários são obrigados a esperar longos períodos para serem atendidos mesmo estando bastante debilitados, além de não receberem informações adequadas na UBS, fatores que acabam se tornando fontes de insatisfação.

Kloetzel et al. ${ }^{13}$ afirmam que o principal motivo da insatisfação de usuários quanto ao serviço oferecido é o tempo de espera muito prolongado e a dificuldade de agendamento de novas consultas. Os pacientes, mesmo aqueles que necessitam de urgência, precisam aguardar por um longo tempo por atendimento ou para marcarem uma consulta, o que determina a insatisfação do paciente. Os autores afirmam também que a satisfação se dá diante de um bom relacionamento com os profissionais de saúde e de aspectos técnicos, como boa infraestrutura e quantidade suficiente de recursos físicos e materiais.

Fatores relacionados aos usuários também devem ser considerados relevantes quando o objetivo é explicar a percepção do serviço oferecido pelo SUS. Gouveia et al..$^{14}$ realizaram estudo a fim de conhecer fatores e aferir diferenças regionais do nível de satisfação dos usuários do sistema de saúde pública brasileiro através da análise dos resultados da Pesquisa Mundial de Saúde com foco na Atenção Básica (PMS-AB) e da Pesquisa 
Mundial de Saúde (PMS). Os autores verificaram que ter baixa escolaridade aumenta as chances de insatisfação com o sistema de saúde brasileiro. $\mathrm{O}$ fato de o indivíduo ter baixa escolaridade está associado a ter um baixo nível de renda, o que implica em pouca ou nenhuma condição de pagar por um serviço privado de saúde. Sendo assim, estes indivíduos acabam sendo usuários exclusivos do SUS, o que aumenta a chance de insatisfação do indivíduo com o sistema. Além disso, estes indivíduos possuem uma autopercepção de saúde ruim.

\section{Metodologia}

Conforme definido anteriormente, este trabalho tem como objetivo explicar o nível de satisfação da população brasileira quanto ao atendimento nas UBS e avaliar as variáveis que o determinam. Como toda avaliação de satisfação, os agentes classificaram sua percepção como ótima, boa, razoável e ruim, ou seja, as opções seguem um ordenamento e diante disso, segundo Pindyck e Rubinfeld ${ }^{15}$, recomenda-se a modelagem probit ordenado para casos em que há um ordenamento, uma hierarquia, das categorias associadas ao fenômeno estudado. É válido citar que este método permite analisar cada determinante que influencia a satisfação do indivíduo ao ser atendido na UBS separadamente segundo o nível de satisfação.

Neste artigo, o nível de satisfação dos usuários foi classificado em quatro níveis: ótimo, bom, regular e ruim. Sendo assim, a variável dependente é discreta e qualitativa, de forma que assume valores que buscam ordenar os dados de forma hierárquica e não linear.

No modelo, são considerados três grupos de variáveis explicativas, sendo que o primeiro grupo $\left(X_{1}\right)$ apresenta variáveis relativas à satisfação e percepção dos cidadãos quanto ao serviço de saúde, utilização e acesso. O segundo grupo $\left(X_{2}\right)$ apresenta variáveis relativas ao processo de trabalho da equipe de profissionais e a organização do cuidado com o usuário. No terceiro grupo $\left(X_{3}\right)$ estão variáveis relativas às condições de infraestrutura, materiais, insumos e medicamentos da unidade. Sendo assim, o modelo é apresentado pela equação (1):

$$
Z_{i}=\beta_{0}+\beta_{1 p} X_{1 p}+\beta_{2 s} X_{2 s}+\beta_{3 t} X_{3 t}+\varepsilon_{\mathrm{i}}
$$

Em que:

$p$ varia de 1 a 16 representando a quantidade de parâmetros para o primeiro grupo de variáveis; $s$ varia de 17 a 21, indicando a quantidade de parâmetros para o segundo grupo de variáveis

$t$ varia de 22 a 27 mostrando a quantidade de parâmetros para o terceiro grupo de variáveis.

$X_{1 p}, X_{2 s}$ e $X_{3 t}$ são matrizes contendo dados das variáveis dos respectivos grupos.

Os três grupos de variáveis são descritos nos Quadros 1, 2 e 3, respectivamente.

O fato de $68 \%$ dos usuários da amostra não terem suas rendas informadas impossibilitou a utilização da variável de salário. Sendo assim, utilizou-se as variáveis Bolsa Família e Ocupado como proxies da situação econômica dos indivíduos e seu efeito no nível de satisfação. A escolha das demais variáveis foi realizada com base na literatura acerca do tema, discutido anteriormente.

Espera-se que variáveis relacionadas aos usuários dos serviços das UBSs impactem positivamente o nível de satisfação, com exceção da variável referente aos dados do Tempo de Espera. O mesmo é esperado para as variáveis referentes aos dados de equipe de trabalho e infraestrutura das UBSs.

Na equação (2), $Z_{i}$ é uma variável latente não observada que informa o nível de satisfação relacionado ao atendimento nas UBS de cada indivíduo, logo tem-se que:

$$
Y_{i}=\left\{\begin{array}{lr}
1, \text { se } \quad Z_{i} \leq \mu_{1} \\
\text { 2, se } \mu_{1}<Z_{i} \leq \mu_{2} \\
\text { 3, se } \mu_{2}<Z_{i} \leq \mu_{3} \\
\text { 4, se } \quad \mu_{3} \leq Z_{i}
\end{array}\right.
$$

Onde $\mu_{1}, \mu_{2}$ e $\mu_{3}$ são limites não observados. No caso específico deste artigo, a variável dependente capta o nível de satisfação do indivíduo em relação ao atendimento nas UBS, sendo $Y_{i}=$ 1 para muito bom, $Y_{i}=2$ para bom, $Y_{i}=3$ para regular e $Y_{i}=4$ para ruim. É importante ressaltar que a categoria 4 é junção de outras duas: ruim e muito ruim. $O$ termo $\varepsilon_{\mathrm{i}}$ possui distribuição normal, com média igual a zero e variância igual a um. O indivíduo que define seu nível de satisfação com o atendimento das UBS como muito bom $\left(Y_{i}=1\right)$ é classificado na categoria $Z_{i} \leq \mu_{1}$.

$$
\begin{gathered}
\operatorname{Pr}\left(Y_{i}=1 / X_{1}, X_{2}, X_{3}\right)=\operatorname{Pr}\left(\mathrm{Z}_{i} \leq \mu_{1}\right) \\
\operatorname{Pr}\left(Y_{i}=1 / X_{1}, X_{2}, X_{3}\right)=\operatorname{Pr}\left(\beta_{0}+\beta_{1 i} X_{1}\right. \\
\left.+\beta_{2 j} X_{2}+\beta_{3 k} X_{3}+\varepsilon_{\mathrm{i}} \leq \mu_{1}\right) \\
\operatorname{Pr}\left(Y_{i}=1 / X_{1}, X_{2}, X_{3}\right)=\operatorname{Pr}\left(\mu_{1}-\beta_{1 i} X_{1}\right. \\
\left.\quad-\beta_{2 j} X_{2}-\beta_{3 k} X_{3} \geq \varepsilon_{\mathrm{i}}\right) \\
\operatorname{Pr}\left(Y_{i}=1 / X_{1}, X_{2}, X_{3}\right)=\mathrm{F}\left(\mu_{1}-\left(\beta_{0}+\beta_{1 i} X_{1}\right.\right. \\
\left.\left.+\beta_{2 j} X_{2}+\beta_{3 k} X_{3}\right)\right)
\end{gathered}
$$


Quadro 1. Descrição das variáveis relacionadas aos usuários dos serviços das UBSs.

\begin{tabular}{|c|c|c|}
\hline \multicolumn{3}{|c|}{ Satisfação e percepção dos usuários quanto ao serviço de saúde, utilização e acesso $\left(X_{1 p}\right)$} \\
\hline Dados & Variáveis & Descrição das variáveis \\
\hline Sexo do indivíduo. & Sexo & $\begin{array}{l}\text { Dummy que representa o sexo do indivíduo } \\
\text { entrevistado. Assume valor } 1 \text { para masculino e } \\
\text { valor } 0 \text { para feminino. }\end{array}$ \\
\hline Cor do indivíduo. & Cor ou raça & $\begin{array}{l}\text { Dummy que representa a cor do indivíduo. } \\
\text { Assume valor } 1 \text { para branco e } 0 \text { para não branco. }\end{array}$ \\
\hline Grau de escolaridade. & $\begin{array}{l}\text { Ensino fundamental } \\
\text { completo, ensino médio } \\
\text { completo, ensino } \\
\text { superior completo } \\
\end{array}$ & $\begin{array}{l}\text { Dummies para representarem o grau de } \\
\text { escolaridade do indivíduo entrevistado. Assume } 1 \\
\text { se o indivíduo possuir o grau de escolaridade em } \\
\text { questão e } 0 \text { caso contrário. }\end{array}$ \\
\hline Se o indivíduo trabalha. & Ocupado & $\begin{array}{l}\text { Dummyque indica se o indivíduo estava } \\
\text { empregado ou não no momento da entrevista. }\end{array}$ \\
\hline $\begin{array}{l}\text { Se o indivíduo recebe benefício } \\
\text { do Bolsa Família. }\end{array}$ & Bolsa Família & $\begin{array}{l}\text { Dummy que assume valor } 1 \text { se o indivíduo receber } \\
\text { benefício do programa e } 0 \text { caso contrário. }\end{array}$ \\
\hline Acesso até a unidade. & $\begin{array}{l}\text { Acesso razoável, acesso } \\
\text { difícil }\end{array}$ & $\begin{array}{l}\text { Dummies que representam como o indivíduo } \\
\text { classifica o acesso até a unidade. Assume valor } 1 \\
\text { em caso afirmativo e } 0 \text { caso contrário. }\end{array}$ \\
\hline $\begin{array}{l}\text { Se o horário de funcionamento } \\
\text { da unidade atende as } \\
\text { necessidades do indivíduo. }\end{array}$ & $\begin{array}{l}\text { Horário de } \\
\text { funcionamento }\end{array}$ & $\begin{array}{l}\text { Dummy que assume valor } 1 \text { se o horário } \\
\text { de funcionamento da unidade atender as } \\
\text { necessidades do indivíduo e } 0 \text { caso contrário. }\end{array}$ \\
\hline $\begin{array}{l}\text { Se a unidade marca consulta via } \\
\text { telefone. }\end{array}$ & Marcar por telefone & $\begin{array}{l}\text { Dummy assume valor } 1 \text { se há marcação de } \\
\text { consulta por telefone e } 0 \text { caso contrário. }\end{array}$ \\
\hline $\begin{array}{l}\text { Tempo de espera para ser } \\
\text { atendido por um profissional } \\
\text { de saúde. }\end{array}$ & $\begin{array}{l}\text { 1h, 1h30min, 2h, } \\
\text { 2h30min, 3h, 2h30min, } \\
\text { 3h, 3h30min, mais de } 4 \mathrm{~h}\end{array}$ & $\begin{array}{l}\text { Dummies que representam o tempo de espera por } \\
\text { atendimento do indivíduo. Assumem valor } 1 \mathrm{em} \\
\text { caso afirmativo e } 0 \text { caso contrário. }\end{array}$ \\
\hline Se há privacidade no consultório & Privacidade & $\begin{array}{l}\text { Dummy que assume valor } 1 \text { caso o indivíduo } \\
\text { julgue que há privacidade no consultório e } 0 \text { caso } \\
\text { contrário. }\end{array}$ \\
\hline $\begin{array}{l}\text { Se os profissionais orientam na } \\
\text { recuperação. }\end{array}$ & $\begin{array}{l}\text { Orientação dos } \\
\text { profissionais. }\end{array}$ & $\begin{array}{l}\text { Dummy que assume valor } 1 \text { se há orientação } \\
\text { profissional na recuperação do paciente e } 0 \text { caso } \\
\text { contrário. }\end{array}$ \\
\hline $\begin{array}{l}\text { Se o indivíduo se sente } \\
\text { respeitado ao ser atendido na } \\
\text { unidade. }\end{array}$ & Respeito & $\begin{array}{l}\text { Dummy que assume valor } 1 \text { caso o indivíduo se } \\
\text { sinta respeitado e } 0 \text { caso contrário. }\end{array}$ \\
\hline $\begin{array}{l}\text { Se os profissionais chamam o } \\
\text { usuário pelo nome. }\end{array}$ & Nome & $\begin{array}{l}\text { Dummy que assume valor } 1 \text { se o indivíduo é } \\
\text { chamado pelo nome e } 0 \text { caso contrário. }\end{array}$ \\
\hline $\begin{array}{l}\text { Se os usuários conseguem } \\
\text { conversar com os profissionais } \\
\text { facilmente. }\end{array}$ & Facilidade & $\begin{array}{l}\text { Dummy que assume valor } 1 \text { caso haja facilidade } \\
\text { para o usuário conversar com profissionais e } 0 \text { caso } \\
\text { contrário. }\end{array}$ \\
\hline $\begin{array}{l}\text { Se um medicamento, quando } \\
\text { receitado, se encontra disponível } \\
\text { na unidade de atendimento. }\end{array}$ & $\begin{array}{l}\text { Disponibilidade de } \\
\text { medicamento }\end{array}$ & $\begin{array}{l}\text { Dummy que assume valor } 1 \text { caso o medicamento se } \\
\text { encontre disponivel na unidade e } 0 \text { caso contrário. }\end{array}$ \\
\hline $\begin{array}{l}\text { Se o indivíduo consegue fazer } \\
\text { reclamação ou sugestão. }\end{array}$ & Reclamação ou sugestão & $\begin{array}{l}\text { Dummy que assume valor } 1 \text { caso se o indivíduo } \\
\text { consegue fazer reclamação ou sugestão e } 0 \text { caso } \\
\text { contrário. }\end{array}$ \\
\hline
\end{tabular}

Fonte: Elaboração a partir dos dados disponibilizados pelo PMAQ (Programa de Melhoria do Acesso e da Qualidade na Atenção Básica).

O mesmo pode ser feito para a opção em que o usuário declararia seu nível de satisfação como bom, sendo definido como:

$$
\begin{gathered}
\operatorname{Pr}\left(Y_{i}=2 / X_{1}, X_{2}, X_{3}\right)=\operatorname{Pr}\left(\mu_{1}<Z_{i} \leq \mu_{2}\right) \\
\operatorname{Pr}\left(Y_{i}=1 / X_{1}, X_{2}, X_{3}\right)= \\
\mathrm{F}\left(\mu_{2}-\left(\beta_{0}+\beta_{1 l} X_{1}+\beta_{2 j} X_{2}+\beta_{3 k} X_{3}\right)\right) \\
-\mathrm{F}\left(\mu_{1}-\left(\beta_{0}+\beta_{1 l} X_{1}+\beta_{2 j} X_{2}+\beta_{3 k} X_{3}\right)\right)
\end{gathered}
$$


Quadro 2. Descrição das variáveis relacionadas à equipe de trabalho das UBSs.

\begin{tabular}{|c|c|c|}
\hline \multicolumn{3}{|c|}{ Processo de trabalho da equipe e organização do cuidado $\left(\mathrm{X}_{2 \mathrm{~s}}\right)$} \\
\hline Dados & Variáveis & Descrição das variáveis \\
\hline $\begin{array}{l}\text { Se a equipe dispõe de canais de } \\
\text { comunicação com os usuários }\end{array}$ & $\begin{array}{l}\text { Comunicação com } \\
\text { usuários. }\end{array}$ & $\begin{array}{l}\text { Dummy que assume valor } 1 \text { caso haja canais de } \\
\text { comunicação com usuários e } 0 \text { caso contrário. }\end{array}$ \\
\hline $\begin{array}{l}\text { Oferta de ações de educação } \\
\text { organizadas pela gestão municipal. }\end{array}$ & Ações de educação & $\begin{array}{l}\text { Dummy que assume valor } 1 \text { caso haja na } \\
\text { unidade ações de educação organizadas pelo } \\
\text { município e } 0 \text { caso contrário. }\end{array}$ \\
\hline $\begin{array}{l}\text { Quantos dias por semana ocorre o } \\
\text { atendimento na unidade de saúde. }\end{array}$ & Dias da semana. & $\begin{array}{l}\text { Dummy que assume valor } 1 \text { caso o atendimento } \\
\text { ocorra até } 3 \text { dias por semana e } 0 \text { caso ocorra de } \\
\text { quatro a mais de cinco dias por semana. }\end{array}$ \\
\hline $\begin{array}{l}\text { Se o indivíduo consegue sair da } \\
\text { unidade com consulta marcada } \\
\text { para o mesmo dia em que procurou } \\
\text { atendimento. }\end{array}$ & $\begin{array}{l}\text { Consulta marcada para } \\
\text { o mesmo dia }\end{array}$ & $\begin{array}{l}\text { Dummy que assume valor } 1 \text { caso o indivíduo } \\
\text { consiga marcar consulta para o mesmo dia e } 0 \\
\text { caso contrário. }\end{array}$ \\
\hline $\begin{array}{l}\text { Se na unidade há mecanismo de } \\
\text { avaliação de satisfação dos usuários. }\end{array}$ & Avaliação da satisfação & $\begin{array}{l}\text { Dummy que assume valor } 1 \text { caso haja } \\
\text { mecanismo de avaliação da satisfação e } 0 \text { caso } \\
\text { contrário. }\end{array}$ \\
\hline
\end{tabular}

Fonte: Elaboração a partir de dados disponibilizados pelo PMAQ (Programa de Melhoria do Acesso e da Qualidade na Atenção Básica).

Quadro 3. Descrição das variáveis relativas à infraestrutura das UBSs.

\begin{tabular}{|l|l|l|}
\hline \multicolumn{2}{|c|}{ Condições de infraestrutura, materiais, insumos e medicamentos da unidade $\left(\mathrm{X}_{3+}\right)$} \\
\hline $\begin{array}{l}\text { Se a unidade divulga lista das } \\
\text { ações e serviços oferecidos. }\end{array}$ & $\begin{array}{l}\text { Variáveis } \\
\text { sista de ações e } \\
\text { serviços }\end{array}$ & $\begin{array}{l}\text { Dummy que assume valor } 1 \text { caso a unidade divulga lista } \\
\text { de ações e serviços oferecidos e 0 caso contrário. }\end{array}$ \\
\hline $\begin{array}{l}\text { Se a unidade divulga escala de } \\
\text { profissionais. }\end{array}$ & $\begin{array}{l}\text { Escala de } \\
\text { profissionais }\end{array}$ & $\begin{array}{l}\text { Dummy que assume valor } 1 \text { caso a unidade divulgue } \\
\text { escala de profissionais e 0 caso contrário. }\end{array}$ \\
\hline $\begin{array}{l}\text { Se há sala de recepção e espera na } \\
\text { unidade. }\end{array}$ & $\begin{array}{l}\text { Sala de recepção } \\
\text { e espera }\end{array}$ & $\begin{array}{l}\text { Dummy que assume valor } 1 \text { caso haja sala de recepção e } \\
\text { espera na unidade e 0 caso contrário. }\end{array}$ \\
\hline $\begin{array}{l}\text { Se há sanitários disponíveis para } \\
\text { usuários na unidade. }\end{array}$ & Sanitários & $\begin{array}{l}\text { Dummy que assume valor } 1 \text { caso haja banheiro } \\
\text { disponível para usuários na unidade e 0 caso contrário. }\end{array}$ \\
\hline $\begin{array}{l}\text { Se existe consultórios particulares } \\
\text { para médicos na unidade. }\end{array}$ & $\begin{array}{l}\text { Consultórios } \\
\text { médicos }\end{array}$ & $\begin{array}{l}\text { Dummy que assume valor } 1 \text { se há consultórios para } \\
\text { médicos na unidade e 0 caso contrário. }\end{array}$ \\
\hline Se há sala de vacina na unidade. & Sala de vacina & $\begin{array}{l}\text { Dummy que assume valor } 1 \text { caso haja sala de vacina na } \\
\text { unidade e 0 caso contrário. }\end{array}$ \\
\hline
\end{tabular}

Fonte: Elaboração a partir de dados disponibilizados pelo PMAQ (Programa de Melhoria do Acesso e da Qualidade na Atenção Básica).

Onde $F($.) é a função de distribuição normal acumulada e $X_{1}, X_{2}$ e $X_{3}$ representam as matrizes de variáveis selecionadas para o estudo. Cálculos de probabilidade semelhantes podem ser definidos para as condições regular e ruim.

A base de dados utilizada no presente artigo é composta pelos microdados de avaliação externa, do $2^{\circ}$ ciclo, de 2013, do Programa Nacional de Melhoria do Acesso e da Qualidade da Atenção Básica $(\mathrm{PMAQ})^{16}$ do Departamento de Atenção Básica (DAB) do Ministério da Saúde.
O PMAQ foi iniciado em 2011 com o objetivo de incentivar funcionários e gestores a melhorar a qualidade dos serviços de saúde oferecidos à população. Para isso, o programa adota um conjunto de estratégias de qualificação, acompanhamento e avaliação do trabalho das equipes de saúde. Uma das vantagens do programa é o aumento do repasse de recursos financeiros do governo federal para os municípios que são bem avaliados. Os microdados de avaliação externa contêm dados sobre a infraestrutura das 
UBS, dados sobre a equipe de profissionais e dados sobre os usuários dessas unidades, sendo que os municípios foram estratificados por um índice construído a partir de aspectos sociais, demográficos e econômicos, como demonstrado no manual de instrução do PMAQ do DAB de 2013.

$\mathrm{O}$ índice foi construído levando em consideração: Produto Interno Bruto (PIB) per capita; percentual da população com plano de saúde; percentual da população com Bolsa Família; percentual da população em extrema pobreza; e, densidade demográfica. Os dados do PIB per capita e da densidade demográfica foram disponibilizados pelo IBGE tendo o ano de 2008 como base. Já os dados de plano de saúde, por município, são do ano de 2009 e disponibilizados pela Agência Nacional de Saúde (ANS). Já o percentual de população em extrema pobreza foi calculado pelo IBGE, com base no universo preliminar do censo demográfico de 2010, enquanto que, o percentual da população com Bolsa Família foi informado pelo Ministério de Desenvolvimento e Combate à Fome, também com o ano de 2010 como referência. Sendo assim, foram construídos seis estratos, em que o PIB per capita tem peso 2, e os demais componentes tem peso 1 , de acordo com a pontuação do índice e porte populacional. O Quadro 4 demonstra como foi criado cada estrato.

\section{Análise do nível de satisfação no atendimento das UBS}

Inicialmente é importante ressaltar que indivíduos que não responderam alguma pergunta e aqueles cuja resposta foi "não sei" ou "não lembra" em relação a qualquer variável utilizada foram excluídos da amostra, restando 35.286 indivíduos usuários de unidades básicas de saúde e aproximadamente 11.700 UBSs, no ano de 2013.
Na Tabela 1 são apresentadas as características da amostra e de seus respectivos níveis de satisfação com o sistema de saúde pública no Brasil em 2013.

Após a estimação do probit ordenado foi possível observar as probabilidades associadas a cada nível de satisfação no atendimento das UBS de um indivíduo médio, isto é, uma mulher não branca, que trabalha, espera cerca de 30 minutos para ser atendido por um profissional de saúde, não recebe benefício do programa Bolsa Família, tem fácil acesso até a unidade e ensino médio completo. A probabilidade de um indivíduo com estas características ter o nível de satisfação regular é de 7,44\% e de ter o nível de satisfação ruim é de 1,06\%. Já em relação aos níveis ótimo e bom, a probabilidade de o indivíduo médio ter esses níveis de satisfação em relação ao atendimento nas UBS é igual a $32,00 \%$ e 59,48\%, respectivamente.

A Tabela 2 apresenta o modelo para os determinantes da satisfação dos indivíduos no atendimento das UBS. Pode-se observar que as variáveis selecionadas para a análise apresentaram, em sua maioria, resultados conforme a literatura e que a maioria delas foram estatisticamente significativas. Antes de analisar o resultado da estimação, é importante lembrar que a variável dependente, ou seja, o nível de satisfação dos usuários está definido em ordem decrescente: ótimo $=1$, bom $=$ 2 , regular $=3$ e ruim $=4$.

Como é possível observar, a maioria das variáveis do grupo 1 foram estatisticamente significativas, com exceção das variáveis sexo e acesso difícil à UBS. Acredita-se que a não significância da variável "sexo" pode estar relacionada à socialização masculina, na qual o cuidado é visto como uma prática feminina, causando a predominância feminina na procura por serviços de saúde, principalmente nos serviços de atenção primária $^{17}$.

Quadro 4. Estratificação dos Municípios de acordo com sua pontuação total e porte populacional.

\begin{tabular}{|c|l|}
\hline Estrato & \multicolumn{1}{c|}{ Critérios de Estratificação } \\
\hline $\mathbf{1}$ & Municípios com pontuação menor que 4,82 e população de até 10 mil habitantes. \\
\hline $\mathbf{2}$ & Municípios com pontuação menor que 4,82 e população de até 20 mil habitantes. \\
\hline $\mathbf{3}$ & Municípios com pontuação menor que 4,82 e população de até 50 mil habitantes \\
\hline $\mathbf{4}$ & $\begin{array}{l}\text { Municípios com pontuação entre 4,82 e 5,4 e população de até } 100 \text { mil habitantes; e municípios com } \\
\text { pontuação menor que 4,82 e população entre 50 e } 100 \text { mil habitantes }\end{array}$ \\
\hline $\mathbf{5}$ & $\begin{array}{l}\text { Municípios com pontuação entre 5,4 e 5,85 e população de até } 500 \text { mil habitantes; e municípios com } \\
\text { pontuação menor que 5,4 e população entre } 100 \text { e } 500 \text { mil habitantes }\end{array}$ \\
\hline $\mathbf{6}$ & Municípios com população acima de 500 mil habitantes ou com pontuação igual ou superior a 5,85. \\
\hline
\end{tabular}


Tabela 1. Características selecionadas de acordo com o grau de satisfação no atendimento das UBS, segundo dados do PMAQ 2013.

\begin{tabular}{|c|c|c|c|c|c|c|}
\hline & & & Nível de s & tisfação & & \\
\hline Caracter & ísticas & Ótimo & Bom & Regular & Ruim & $\begin{array}{l}\text { Total das } \\
\text { variáveis }\end{array}$ \\
\hline Sexo & Masculino & $34,80 \%$ & $54,40 \%$ & $7,54 \%$ & $3,26 \%$ & $17,47 \%$ \\
\hline & Feminino & $31,96 \%$ & $52,14 \%$ & $10,71 \%$ & $5,19 \%$ & $82,53 \%$ \\
\hline Cor & Branco & $35,29 \%$ & $51,69 \%$ & $8,70 \%$ & $4,33 \%$ & $42,44 \%$ \\
\hline & Não Branco & $30,36 \%$ & $53,16 \%$ & $11,24 \%$ & $5,24 \%$ & $57,56 \%$ \\
\hline Bolsa Família & Sim & $26,99 \%$ & $54,99 \%$ & $12,37 \%$ & $5,65 \%$ & $33,72 \%$ \\
\hline & Não & $35,23 \%$ & $51,28 \%$ & $9,04 \%$ & $4,45 \%$ & $66,28 \%$ \\
\hline Grau de Escolaridade & Fundamental incompleto & $33,07 \%$ & $53,78 \%$ & $8,57 \%$ & $4,57 \%$ & $47,70 \%$ \\
\hline & Fundamental completo & $30,11 \%$ & $52,68 \%$ & $11,60 \%$ & $5,61 \%$ & $7,68 \%$ \\
\hline & Médio completo & $31,63 \%$ & $51,28 \%$ & $12,04 \%$ & $5,05 \%$ & $26,30 \%$ \\
\hline & Superior completo & $42,63 \%$ & $45,52 \%$ & $8,96 \%$ & $2,90 \%$ & $4,21 \%$ \\
\hline Acesso até a unidade & Fácil & $34,69 \%$ & $52,45 \%$ & $8,78 \%$ & $4,08 \%$ & $80,30 \%$ \\
\hline & Razoável & $23,41 \%$ & $53,01 \%$ & $16,74 \%$ & $6,84 \%$ & $11,81 \%$ \\
\hline & Difícil & $26,23 \%$ & $47,06 \%$ & $13,24 \%$ & $13,48 \%$ & $1,16 \%$ \\
\hline Ocupado & Sim & $32,21 \%$ & $52,38 \%$ & $10,44 \%$ & $4,97 \%$ & $36,04 \%$ \\
\hline & Não & $32,59 \%$ & $52,62 \%$ & $10,00 \%$ & $4,79 \%$ & $63,96 \%$ \\
\hline Mecanismo de avaliação & $\operatorname{Sim}$ & $34,40 \%$ & $51,66 \%$ & $9,68 \%$ & $4,27 \%$ & $73,39 \%$ \\
\hline dos usuários & Não & $27,30 \%$ & $54,96 \%$ & $11,37 \%$ & $6,38 \%$ & $26,61 \%$ \\
\hline Quantidade de dias na & Até 3 dias por semana & $27,58 \%$ & $56,91 \%$ & $10,53 \%$ & $4,97 \%$ & $8,68 \%$ \\
\hline $\begin{array}{l}\text { semana em que ocorre o } \\
\text { atendimento }\end{array}$ & Mais de 3 dias por semana & $32,98 \%$ & $52,12 \%$ & $10,09 \%$ & $4,81 \%$ & $91,32 \%$ \\
\hline Há sala de recepção e espera & Sim & $32,52 \%$ & $52,53 \%$ & $10,11 \%$ & $4,83 \%$ & $97,70 \%$ \\
\hline na unidade & Não & $30,52 \%$ & $52,53 \%$ & $11,79 \%$ & $5,46 \%$ & $2,30 \%$ \\
\hline Há sanitários para os & Sim & $32,52 \%$ & $52,47 \%$ & $10,15 \%$ & $4,86 \%$ & $97,99 \%$ \\
\hline usuários & Não & $30,59 \%$ & $55,10 \%$ & $10,48 \%$ & $3,82 \%$ & $2,01 \%$ \\
\hline Total da amostra & & $32,45 \%$ & $52,53 \%$ & $10,16 \%$ & $4,85 \%$ & \\
\hline
\end{tabular}

Fonte: Elaboração a partir dos dados disponibilizados pelo PMAQ (Programa de Melhoria do Acesso e da Qualidade na Atenção Básica).

Além disso, na Tabela 2 é possível verificar que a amostra é composta predominantemente por pessoas do gênero feminino. $O$ fato do acesso não ter afetado o nível de satisfação explica-se pelo fato de as UBSs estarem estrategicamente localizadas. Um indicativo disso, quando analisada a Tabela 2, seria o fato de que apenas 1,1\% dos usuários classificariam o acesso à unidade como difícil.

A cor do indivíduo apresentou relação negativa com o nível de satisfação. De acordo com os resultados, o indivíduo branco tende a ter melhor percepção do atendimento das unidades básicas. Ribeiro et al. ${ }^{18}$ relacionaria esse fenômeno com a constatação de que essa parcela da população estaria correlacionada com níveis mais altos de renda, tornando-os mais independentes do sistema público, apresentando, portanto, maior propensão à satisfação com o serviço público de saúde uma vez que poderiam estar utilizando tal serviço por opção.

As variáveis Ensino Fundamental Completo e Ensino Médio Completo apresentaram relação positiva com o nível de satisfação, enquanto que a variável Ensino Superior a apresentou negativa. Estes resultados mostram que o indivíduo com menor grau de escolaridade tende a ter pior nível de satisfação em relação ao atendimento na UBS devido ao fato de que quanto menor o grau de escolaridade, pior é a percepção de que os serviços prestados pelas UBSs são ótimos ou bons. De fato, na Tabela 2, pode-se perceber que o percentual de indivíduos com menor grau de satisfação é maior para aqueles com menor grau de escolaridade e que indivíduos com ensino superior completo constituem uma parte ínfima da amostra.

Indivíduos economicamente ocupados estariam relacionados de forma positiva com a per- 
Tabela 2. Resultados do modelo estimado para determinar o nível de satisfação no atendimento das UBS.

\begin{tabular}{|c|c|c|c|}
\hline Variáveis explicativas & Coeficiente & $\begin{array}{c}\text { Desvio padrão } \\
\text { (robustos) }\end{array}$ & P-valor \\
\hline Sexo & $-0,0175691$ & 0,0168084 & 0,296 \\
\hline Cor & $-0,0688052$ & 0,0128303 & 0,000 \\
\hline Ensino fundamental completo & 0,1110385 & 0,0159933 & 0,000 \\
\hline Ensino médio completo & 0,1094303 & 0,0154419 & 0,000 \\
\hline Ensino superior completo & $-0,0944521$ & 0,0330552 & 0,004 \\
\hline Ocupação & 0,0402708 & 0,0134163 & 0,003 \\
\hline Bolsa Família & 0,1552553 & 0,0136747 & 0,000 \\
\hline Acesso razoável & 0,1858997 & 0,0191768 & 0,000 \\
\hline Acesso difícil & 0,0177622 & 0,0642378 & 0,782 \\
\hline Horário de funcionamento & $-0,3831477$ & 0,0209111 & 0,000 \\
\hline Marcação por telefone & $-0,1205012$ & 0,0234418 & 0,000 \\
\hline 1h de espera & 0,2867334 & 0,0151102 & 0,000 \\
\hline 1h30min de espera & 0,3684025 & 0,0278891 & 0,000 \\
\hline $2 \mathrm{~h}$ de espera & 0,45408 & 0,0222562 & 0,000 \\
\hline 2h30min de espera & 0,5297564 & 0,0485037 & 0,000 \\
\hline $3 \mathrm{~h}$ de espera & 0,5591771 & 0,0359513 & 0,000 \\
\hline 3h30min de espera & 0,7596442 & 0,0781824 & 0,000 \\
\hline 4h de espera & 0,6750433 & 0,0662677 & 0,000 \\
\hline Mais de $4 \mathrm{~h}$ de espera & 0,6662699 & 0,6662699 & 0,000 \\
\hline Privacidade & $-0,2401566$ & 0,0317687 & 0,000 \\
\hline Orientação & $-0,2156478$ & 0,0244737 & 0,000 \\
\hline Respeito & $-0,4690991$ & 0,0419077 & 0,000 \\
\hline Nome & $-0,2982874$ & 0,0294662 & 0,000 \\
\hline Facilidade & $-0,2228022$ & 0,0134529 & 0,000 \\
\hline Disponibilidade de medicamento & $-0,0985587$ & 0,0136593 & 0,000 \\
\hline Reclamação ou sugestão & 0,1086447 & 0,0151924 & 0,000 \\
\hline Ações de educação & $-0,0007146$ & 0,0207286 & 0,972 \\
\hline Dias da semana & 0,0323585 & 0,0218852 & 0,139 \\
\hline Consulta marcada para o mesmo dia & $-0,0368323$ & 0,0234723 & 0,117 \\
\hline Avaliação da satisfação & $-0,067477$ & 0,0169979 & 0,000 \\
\hline Comunicação com usuários & $-0,0348476$ & 0,0204604 & 0,089 \\
\hline Lista de ações e serviços & $-0,0248126$ & 0,0166889 & 0,137 \\
\hline Escala de profissionais & $-0,0613864$ & 0,0144788 & 0,000 \\
\hline Sala de recepção e espera & 0,0038056 & 0,0417949 & 0,927 \\
\hline Sanitários & 0,0059828 & 0,044457 & 0,893 \\
\hline Consultórios médicos & $-6.40 e-06$ & 0,0000372 & 0,863 \\
\hline Sala de vacina & 0,1416687 & 0,0176989 & 0,000 \\
\hline /cut1 & $-0,6786645$ & 0,0930087 & \\
\hline /cut2 & 1,160251 & 0,0933694 & \\
\hline /cut3 & 2,091043 & 0,094262 & \\
\hline
\end{tabular}

Fonte: Resultados da pesquisa.

cepção de satisfação do atendimento das UBS. Ou seja, o indivíduo que trabalha tende a ter uma pior percepção do serviço prestado. Tal fenômeno também pode ser observado na Tabela 2. É válido lembrar que o fato de o indivíduo trabalhar não significa que ele não seja vulnerável financeiramente, visto que seu rendimento pode ser baixo. Com relação à vulnerabilidade financeira, captada pela variável bolsa família, percebe-se uma relação positiva com o nível de satisfação, 
indicando que, novamente, indivíduos vulneráveis financeiramente tendem a estar correlacionados com um pior nível de satisfação.

Os resultados acima podem ser corroborados através do estudo realizado por Bastos e Fasolo ${ }^{19}$. Segundo estes autores, indivíduos vulneráveis financeiramente possuem maior dependência dos serviços públicos de saúde e, devido a essa dependência, apresentam menor nível de satisfação em relação ao atendimento nas UBS. Os indivíduos com maior renda, apesar de também utilizarem o serviço de saúde pública, têm condições de buscarem serviços de saúde privados ou particulares caso se sintam insatisfeitos e só os utilizariam em condições favoráveis.

Com relação ao acesso, a variável Acesso Razoável apresentou impacto negativo no nível de satisfação, ou seja, indivíduos que classificaram o acesso até a unidade como razoável tendem a se sentirem menos satisfeitos com o atendimento. Segundo Jorge e Salete $\mathrm{e}^{20}$ a melhora em fatores ambientais como o acesso até a unidade tem impacto positivo no nível de satisfação do usuário. Da mesma forma, o horário de funcionamento apresentou impacto positivo no nível de satisfação. De acordo com os resultados, o indivíduo que julga ter suas necessidades atendidas com o horário de funcionamento tem maior nível de satisfação.

De igual maneira, a marcação de consulta por telefone apresentou impacto positivo no nível de satisfação, indicando que quando a unidade realiza marcação de consulta via telefone, o indivíduo tende a ter um melhor nível de satisfação. Este resultado está de acordo com o esperado e pode ser explicado pelo estudo de Kloetzel et al. ${ }^{13}$. $\mathrm{O}$ indivíduo, muitas vezes, está muito debilitado e não possui condições de ir até a unidade marcar uma consulta, sendo assim, o fato de não ser possível marcar consulta por telefone reduziria sua percepção em termos de satisfação com o atendimento oferecido pelas UBSs.

O tempo de espera para o indivíduo ser atendido por um profissional de saúde apresentou sinal positivo, ou seja, impactou negativamente no nível de satisfação. Percebe-se que todos os coeficientes relacionados com a variável tempo de espera, representada por oito dummies, foram positivos. Entretanto, o valor dos coeficientes cai à medida que o tempo de espera reduz. Muitas vezes, usuários são obrigados a esperar longos períodos para serem atendidos, mesmo estando muito doentes e quanto maior o tempo de espera para atendimento maior o tempo que demora para ter seu problema resolvido.
Quando há privacidade no consultório utilizado para atendimento, de acordo com a percepção do indivíduo, o nível de satisfação é impactado positivamente. Da mesma maneira, o fato de haver orientação dos profissionais quanto à recuperação também apresentou relação negativa sugerindo uma correlação positiva com o nível de satisfação. Essas variáveis fazem parte do que é conhecido por desemprenho profissional e resolutividade. Segundo Moimaz et al. ${ }^{10}$, o desemprenho profissional e a resolutividade impactam positivamente o nível de satisfação dos usuários. $\mathrm{Ou}$ seja, quando a equipe de profissionais tem um bom desempenho, auxiliando na resolução do problema do usuário, este tende a ter um melhor nível de satisfação.

O indivíduo não se sentir respeitado ao ser atendido influenciou positivamente a percepção de satisfação assim como o fato do indivíduo ser chamado pelo nome pelo profissional de saúde. Essas variáveis constituem o aspecto humanitário da relação médico-paciente. De acordo com Espiridião e Trad ${ }^{6}$, quando há uma relação médico-paciente mais humana, os usuários se sentem mais confortáveis e mais confiantes para falar sobre seus problemas com os profissionais de saúde, além de, muitas vezes, perceberem que isso facilita a resolutividade de seus problemas. Sendo assim, os indivíduos tendem a se sentir mais satisfeitos.

Em relação à dummy Facilidade foi observado que quando há facilidade para falar com os profissionais de saúde o nível de satisfação se eleva com resultado similar quando o usuário se depara com a situação onde os medicamentos receitados se encontram disponíveis na UBS.

A dificuldade de expor suas reclamações e propor sugestões para a melhoria do serviço de saúde estaria negativamente correlacionada com o nível de satisfação. Quando o indivíduo consegue fazer uma reclamação ou sugestão, na maioria das vezes, ele consegue fazer junto aos atendentes e/ou recepcionistas, que não são as pessoas mais indicadas para a resolução das pendências dos usuários. Sendo assim, eles não teriam suas reclamações ou sugestões atendidas visto que não são escutados por profissionais de saúde ${ }^{21}$.

Quanto às variáveis do grupo 2, que estão relacionadas à equipe de profissionais de saúde, foram em sua maioria não significativas estatisticamente. A variável avaliação da satisfação, que indica se há na unidade algum mecanismo de avaliação do usuário, foi a que apresentou maior significância e também relação negativa com 
o nível de satisfação sugerindo que, quando há algum meio de avaliar a satisfação dos usuários, estes tendem a ter maior nível de satisfação no atendimento. De fato, percebe-se através da descrição da amostra (Tabela 2), que o percentual de indivíduos que classificam o nível de satisfação como ótimo é maior nas unidades onde há mecanismos de avaliação da satisfação do usuário. Com relação à quantidade de dias da semana em que ocorre o atendimento na UBS, a não significância pode ser explicada pelo fato de em quase a totalidade das unidades da amostra, 91,32\%, o atendimento ocorre em mais de 3 dias por semana, o que também pode ser verificado na descrição da amostra. A mesma análise pode ser feita para as demais variáveis deste grupo.

Da mesma forma, dentre as variáveis do grupo 3, que são as relacionadas à infraestrutura e disponibilidade de recursos da unidade, apenas duas foram estatisticamente significativas: escala de profissionais e sala de vacina.

A variável Escala de Profissionais, que mostra se a unidade divulga a escala dos profissionais que realizarão o atendimento, apresentou efeito positivo no nível de satisfação. De acordo com os resultados, quando a unidade divulga a escala de profissionais, os usuários tendem a se sentir mais satisfeitos com o serviço prestado. Porém, a variável sala de vacina apresentou efeito negativo no nível de satisfação. Sendo assim, os resultados mostram que quando há sala de vacina na unidade, o nível de satisfação tende a ser classificado como regular ou ruim, o que pode indicar que a estrutura física destas salas não está em boas condições. A infraestrutura é o primeiro contato do indivíduo com a unidade básica ao procurar atendimento, portanto, se a unidade conta com boa infraestrutura, o indivíduo terá boas expectativas quanto ao atendimento, tendendo a ter um melhor nível de satisfação ${ }^{22}$.

Por fim, a não significância das demais variáveis do grupo 3, como sala de recepção e de espera e sanitários pode ser explicada pelo fato de que quase a totalidade da amostra conta com essa estrutura, aproximadamente $98 \%$, como é possível verificar na Tabela 2 .

\section{Considerações finais}

O presente artigo procurou analisar o nível de satisfação no atendimento das Unidades Básicas de Saúde e seus determinantes. Esta análise é de grande relevância, visto que a satisfação do usuário é um importante instrumento de avaliação dos serviços de saúde oferecidos e, consequentemente, conhecendo estes determinantes é possível saber quais ações direcionar para melhorar a satisfação do serviço prestado. Ressalta-se que com a crise atravessada pela saúde pública, com unidades sobrecarregadas, falhas na infraestrutura e recursos insuficientes, a satisfação do usuário passa a ser uma variável de extrema importância na determinação do bem-estar da população e da qualidade do serviço.

Sendo assim, para o alcance do objetivo proposto, recorreu-se a uma análise probabilística a fim de verificar quais os fatores que determinam a probabilidade da satisfação dos usuários no atendimento das UBS. O estudo foi feito com base nos microdados de avaliação externa disponibilizados pelo Programa Nacional de Melhoria do Acesso e da Qualidade da Atenção Básica (PMAQ), referente ao ano de 2013, e o modelo utilizado foi o probit ordenado.

Os principais resultados indicaram que o sexo do indivíduo não teve influência significativa sobre o nível de satisfação quanto ao atendimento nas UBS. Ou seja, homens e mulheres possuem a mesma probabilidade de terem um nível de satisfação ótimo. Além disso, características referentes à resolutividade do problema, humanização do cuidado e conforto do paciente apresentaram impacto positivo no nível de satisfação. O tempo de espera para ser atendido impactou negativamente no nível de satisfação.

De maneira geral, é possível afirmar que os principais determinantes da satisfação dos usuários das UBS no Brasil estão relacionados à forma como as UBSs são administradas e ao tratamento pessoal oferecido pelas equipes de profissionais de saúde. Isso pode ser constatado quando se observa que o tempo de espera, a privacidade, o respeito por parte da equipe de saúde e o fato de terem um tratamento mais pessoal, sendo chamados pelo nome, foram as principais variáveis que contribuíram para o aumento de satisfação. Em contrapartida, a infraestrutura tem pouco ou nenhum impacto.

Diante disso, pode-se concluir que a melhora na percepção dos usuários dos serviços de saúde no Brasil, prestado pelas UBSs, pode ser alcançada sem que haja um aumento significativo de recursos financeiros. Para isso, basta a melhora na forma e na logística de atendimento e no respeito para com o usuário, ou seja, melhora na gestão. 


\section{Colaboradores}

MAV Gomes trabalhou na introdução, revisão de literatura, resultados e considerações finais; $\mathrm{VO}$ Pinto na formatação final e ajustes para submissão; e FCC Cassuce na orientação e metodologia do trabalho.

\section{Referências}

1. Instituto Brasileiro de Geografia e Estatística (IBGE). Sintese de Indicadores Sociais. Rio de Janeiro: IBGE; 2017. [acessado 2018 Maio 15]. Disponível em: http://www. ibge.gov.br

2. Brasil. Ministério da Saúde (MS). Sistema Único de Saúde. Brasília: MS. [acessado 2018 Maio 15]. Disponível em: http://portalms.saude.gov.br/sistema-unico-de-saude

3. Pegoraro APA. O sistema de saúde no Brasil e nos Estados Unidos: uma análise comparativa. Anais III Fórum Brasileiro de Pós-Graduação em Ciência Política-UFPR, 2013.

4. Akerman M, Nadanovsky P. Avaliação dos Serviços de Saúde - Avaliar o Quê? Cad Saude Publica 1992; 8(4):361-365.

5. Silva LMV, Formigli VLA. Avaliação em saúde: limites e perspectivas. Cad Saude Publica 1994; 10(1):80-91.

6. Espiridião MA, Trad LAB. Avaliação de satisfação de usuários: considerações teórico-conceituais. Cad Saude Publica 2006; 22(6):1267-1276.

7. Vaitsman J, Andrade GRB. Satisfação e responsividade: formas de medir a qualidade e a humanização da assistência à saúde. Cien Saude Colet 2005; 10(3):599613.

8. Williams B. Patient satisfaction: a valid concept? Soc Sci Med 1994; 38(4):509-516.

9. Bernhart MH, Wiadnyana IGP, Wihardjo H, Pohan I. Patient satisfaction in developing countries. Soc Sci Med 1999; 48(8):989-996.

10. Moimaz SAS, Marques JAM, Saliba O, Garbin CAS, Zina LG, Saliba NA. Satisfação e percepção do usuário do SUS sobre o serviço público de saúde. Physis 2010; 20(4):1419-1440.

11. Callou Filho CR, Machado MDFAS, Mesquita CAM, Rocha LK, Rodrigues MVS. Estratégia Saúde da Família: satisfação dos usuários no município de Fortaleza -CE. Saúde e Pesquisa 2017; 10(2):339-346.

12. Sá SLB, Mattos RR, Pinheiro VL, Pequeno LL, Diógenes MAR. Usuário da estratégia de saúde da família: conhecimento e satisfação sobre acolhimento. Revista Brasileira em Promoção da Saúde 2012; 25(Supl. 2):96103.

13. Kloetzel K, Bertoni AM, Irazoqui MC, Campos VPG, Santos RND. Controle de qualidade em atenção primária à saúde. A satisfação do usuário. Cad Saude Publica 1998; 14(3):263-268.
14. Gouveia GC, Souza WVD, Luna CF, Souza-Júnior PRBD, Szwarcwald CL. Satisfação dos usuários do sistema de saúde brasileiro: fatores associados e diferenças regionais. Rev Bras Epidemiol 2009; 12(3):281-296.

15. Pindyck R, Rubinfeld D. Econometria: modelos e previsões. $4^{\mathrm{a}}$ ed. Rio de Janeiro: Campus; 2004.

16. Brasil. Ministério da Saúde (MS), Instituto Brasileiro de Geografia e Estatística (IBGE). Portal do Departamento de Atenção Básica: Programa de Melhoria do Acesso e da Qualidade (PMAQ), 2011. [acessado 2018 Jul 2]. Disponível em: http://dab.saude.gov.br/portaldab/ape_pmaq.php?conteudo=1_ciclo

17. Levorato CD, Mello LMD, Silva ASD, Nunes AA. Fatores associados à procura por serviços de saúde numa perspectiva relacional de gênero. Cien Saude Colet 2014; 19(4):1263-1274.

18. Ribeiro MCSDA, Barata RB, Almeida MFD, Silva ZPD. Perfil sociodemográfico e padrão de utilização de serviços de saúde para usuários e não-usuários do SUS-PNAD 2003. Cien Saude Colet 2006; 11(4):10111022.

19. Bastos GAN, Fasolo LR. Fatores que influenciam a satisfação do paciente ambulatorial em uma população de baixa renda: um estudo de base populacional. Rev Bras Epidemiol 2013; 16(1):114-124.

20. Jorge B, Salete M. Avaliação da qualidade do Programa Saúde da Família no Ceará: a satisfação dos usuários. Revista Baiana de Saúde Pública 2014; 31(2):256.

21. Gerschman S, Veiga L, Guimarães C, Ugá MAD, Portela MC, Vasconcellos MM, Barbosa PR, Lima SML. Estudo de satisfação dos beneficiários de planos de saúde de hospitais filantrópicos. Cien Saude Colet 2007; 12(2):487-500.

22. Ricci NA, Wanderley FDS, Oliveira MSD, Rebelatto JR. O hospital-escola de São Carlos: análise do funcionamento por meio da satisfação dos usuários. Cien Saude Colet 2011; 16(Supl. 1):1125-1134.

Artigo apresentado em 01/04/2019

Aprovado em 02/07/2019

Versão final apresentada em 04/07/2019

Editores-chefes: Romeu Gomes, Antônio Augusto Moura da Silva 